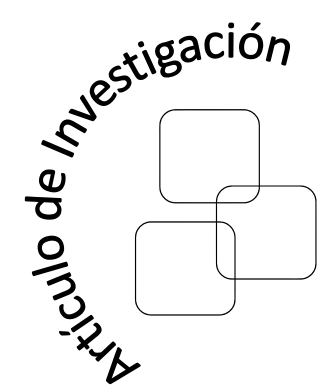

Juan Manuel Aranda Docente investigador Universidad Sergio Arboleda juan.aranda@usa.edu.co

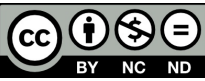

\section{Diseño y evaluación del desempeño de una red de comunicaciones para medición inteligente en Network Simulator-2}

\author{
Design and performance evaluation of a \\ communication network for smart \\ metering in Network Simulator-2
}

\section{Resumen}

Este artículo presenta los resultados de diseño y evaluación del desempeño de una red de comunicaciones para medición inteligente o AMI (Advanced Metering Infrastructure), empleando el simulador de redes Network Simulator-2 (NS-2). La red de comunicaciones propuesta combina las tecnologías PLC (Power Line Communication) y HSDPA (High Speed Downlin Packet Access), así como el uso de concentradores de datos (DC) y de protocolos aplicación y transporte como DLMS/COSEM y TCP-UDP/IP. Los resultados de evaluación indicaron que al introducir dispositivos DC dentro del diseño de red de comunicaciones, ésta presenta un desempeño superior frente a otras que no los emplean para concentrar la información de diferentes medidores inteligentes. Finalmente, se observó una mayor cobertura y un menor número de acceso directos a la red de comunicaciones al considerar DC en el diseño.

Palabras claves: AMI, comunicaciones móviles 3G, DLMS/COSEM, PLC, redes inteligentes.

\section{Abstract}

This article presents the results of design and performance evaluation of a Smart Metering or AMI network, using the Network Simulator-2. The communication network proposed combines technologies such as, PLC and HSPDA, using data concentrators (DCs) and application and transport protocols such as DLMS/COSEM and TCP/IP. The performance evaluation results shown that introducing data concentrator inside a communication network provides a superior performance compared with others that not use it. Finally, the employment of DCs on the design also extended communication coverage and reduce direct accesses to the network.

Key words: AMI, 3G mobile communications, DLMS/COSEM, PLC, Smart Grids. 


\section{Introduction}

Actualmente, las redes eléctricas del mundo están migrando hacia las redes de próxima generación, las cuales soportan el flujo de dos vías (tanto de energía como datos); controlan el consumo de energía de los clientes; permiten localizar, aislar y restaurar rápidamente los puntos donde ocurren cortes de energía; facilitan la integración de generación distribuida a la red de distribución, como un mecanismo para suplir la creciente demanda de energía generada por el advenimiento de las nuevas aplicaciones; y reduce tanto el impacto ambiental producido por las plantas de generación existentes como las pérdidas en la transmisión de la energía. Estas redes conocidas como Smart Grids (redes inteligentes) buscan implementar tres mecanismos clave: (1) eficiencia energética, (2) respuesta de la demanda y (3) control directo sobre la carga [1].

El primer paso de la evolución de las redes eléctricas convencionales a Smart Grids es la implementación de una infraestructura de medición avanzada o AMI (Advanced Metering Infrastructure). AMI hace referencia a un sistema que mide, almacena y analiza la energía utilizada desde dispositivos avanzados, tales como medidores inteligentes (MI), a través de una red de comunicaciones bidireccional implementada sobre diferentes tecnologías. La Figura 1 muestra una arquitectura típica de una red AMI, la cual se compone de tres bloques principales: (1) las unidades de recolección de datos local, (2) la red de comunicaciones y (3) el Centro de Gestión y Control de la compañía de energía.

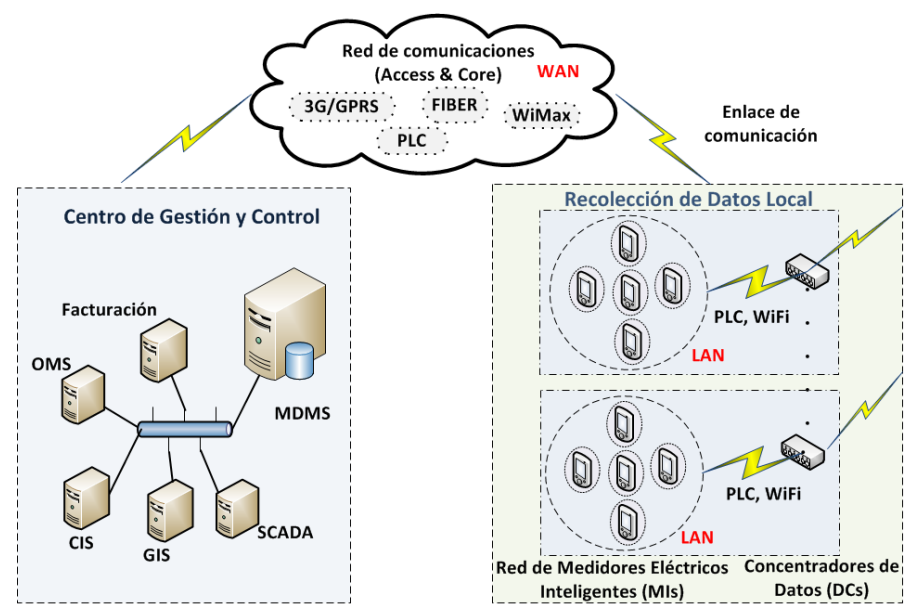

Figura 1. Arquitectura de una red AMI (adaptada de [2]). Se compone principalmente de tres bloques: las unidades de recolección de datos local, la red de comunicaciones y el centro de gestión y control.

De acuerdo con la Figura 1, las redes de comunicaciones constituyen la "columna vertebral" de una arquitectura AMI; por tanto, de la adecuada definición del esquema de comunicaciones dependerá el éxito de la operación de las redes AMI en los Smart Grids.

¿Cuál(es) sería(n) la(s) tecnología(s) y la(s) topología(s) de comunicaciones a implementar en una red AMI? ¿Qué configuración AMI utilizar para lograr interoperabilidad, confiabilidad, rentabilidad y escalabilidad? ¿Cómo aprovechar las infraestructuras eléctricas existentes para transportar al mismo tiempo energía y datos? ¿Cuál sería el impacto del tráfico AMI en 
las redes de telecomunicaciones existentes? ¿Éstas soportarán simultáneamente los servicios de aplicaciones de telefonía móvil/fijo y las aplicaciones AMI? En caso de que se llegara a sobrecargar la red celular, ¿cómo lograr reducir el número de accesos directos a ella, garantizando calidad en los servicios ofrecidos? El presente artículo aborda éstas y otras cuestiones, que surgen a la hora de diseñar o decidir ofrecer servicios de telecomunicaciones a redes AMI.

Revisando la literatura sobre el tema, se encontraron trabajos similares centrados en la evaluación del desempeño de esquemas y protocolos de comunicaciones para redes de lectura de medición o AMR (Automated Meter Reading)/AMI implementados sobre las tecnologías de comunicación a través de las líneas de potencia o PLC (Power Line Communication) [3] y celular [4], y el protocolo DMLS/COSEM (Device Language Message Service/Companion Specification for Energy Metering, protocolo de comunicaciones europeo que permite la interoperabilidad entre los equipos de medición y el centro de recolección de datos en una red AMI). En el primer trabajo revisado [3], se aclara que la implementación del protocolo se centró únicamente en el nivel de aplicación, dejando de lado todo lo relacionado con la capa de transporte COSEM-TCP. Mientras que en el segundo trabajo [4], se implementó la subcapa de transporte COSEM-WRAPPER, pero no hay claridad en la implementación realizada. Adicionalmente, los autores en [4] propusieron un esquema de comunicaciones para una red AMI implementada sobre la tecnología celular de acceso a descarga de paquetes a alta velocidad o HSDPA (High Speed Downlink Packet Access) con capacidad de soportar hasta 130 medidores inteligentes sin afectar los requerimientos de calidad y garantizando factibilidad de interoperabilidad de servicios de acceso a Internet, aplicaciones en tiempo real y servicios AMI compartiendo una misma red de acceso.

Analizando los resultados obtenidos en el segundo trabajo [4], 130 medidores es un número reducido comparado con los miles de medidores que se espera que sean capaces de soportar las redes AMI [5]. Al considerar la tecnología celular para estos tipos de redes, hay que tener en cuenta que los dispositivos de medición requieren múltiples conexiones con la estación base para transferir muy pocos datos. Dado que cada conexión tiene señalización y overheads relativos a los datos, resultan paquetes significativamente grandes comparados con la información transmitida. Por tanto, a medida que se incrementa el número de medidores dentro de la red, podría llegarse a sobrecargar la red celular, limitar la cobertura y afectar la calidad de los servicios prestados, situación que se presentó en el segundo trabajo [4].

El presente artículo va dirigido especialmente a los operadores de red que lideran proyectos de Smart Grids o buscan desplegar una red AMI sobre sus redes eléctricas. Así mismo, está dirigido a los operadores de telefonía móvil interesados en proveer servicios de infraestructura celular requeridos por las aplicaciones AMI, pero que, al mismo tiempo, desean conocer el impacto que conlleva el tráfico AMI a través de sus infraestructuras de telecomunicaciones, de forma que puedan estimar y validar la capacidad de comunicaciones requerida, de acuerdo con distintos parámetros de calidad de servicio y resultados obtenidos por simulación usando distintos escenarios.

Este artículo se encuentra organizado como sigue: la sección 2 presenta el esquema de comunicaciones propuesto para una red AMI, seguido de la descripción del diseño e implementación en NS-2 de los modelos experimentales del protocolo de comunicaciones DLMS/COSEM y del dispositivo concentrador de datos. Posteriormente, en la sección 3 se detallan el escenario de simulación y los requerimientos de telecomunicaciones (métricas) para evaluar el 
desempeño del esquema de comunicaciones, continuando en la sección 4 con el análisis de resultados y finalizando con las conclusiones de la investigación y trabajo futuro en la sección 5.

\section{Red de comunicaciones y modelos experimentales}

\subsection{Esquema de comunicaciones para una red AMI}

El esquema propuesto combina las tecnologías de acceso PLC y HSDPA para establecer una conexión entre una red de medidores inteligentes y el Centro de Control de la compañía de energía. Se compone tanto de medidores inteligentes (MI), incorporados en cada uno de los abonados, como de concentradores de datos (DC), cuyo objetivo es agrupar el tráfico producido por un conjunto de medidores dentro del alcance de su red, implementada sobre la tecnología NB-PLC (Narrow Band-PLC), y de enviarlas hacia el Centro de Control cuando éste los solicite. Entre el concentrador de datos y el Centro de Control se implementa una red de acceso HSDPA y una red de transporte con cobertura metropolitana sobre fibra óptica. La arquitectura de telecomunicaciones, empleada para transferir los paquetes a los diferentes servidores dentro de las instalaciones del Centro de Control, consiste en una red de área local Ethernet.

Con el fin de garantizar la interoperabilidad entre los equipos de medición y de concentración de datos, se propone el protocolo DLMS/COSEM, muy utilizado en proyectos europeos de medición avanzada [5]. Para la comunicación entre el Centro de Control y los DCs se propone una aplicación RequestingAPP (Figura 2), implementada sobre los protocolos TCPUDP/IP y diseñada para correr programas de respuesta de la demanda y aplicaciones de medición avanzada. La Figura 2 presenta la pila de protocolos de los nodos principales que conforman el esquema de comunicaciones para una red AMI implementada en NS-2 ${ }^{1}$.

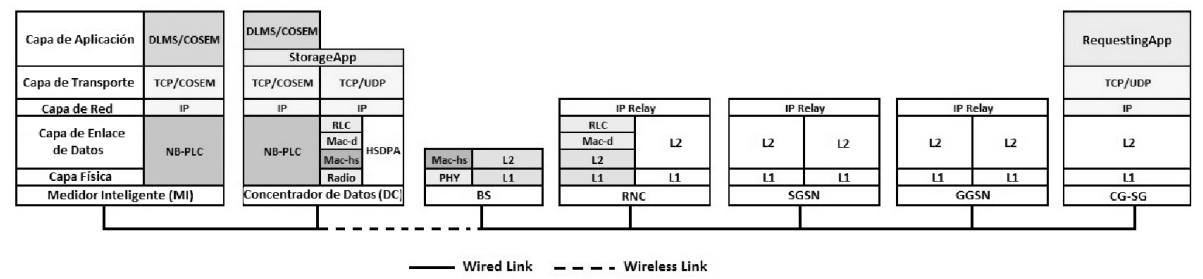

Figura 2. Pila de protocolos: nodos principales del modelo de simulación en NS-2 para el esquema de comunicaciones AMI.

\subsection{Protocolo de comunicaciones DLMS/COSEM}

El protocolo de comunicaciones DLMS/COSEM es un modelo de comunicación que opera bajo el esquema cliente/servidor, donde el MI desempeña el papel de servidor y el DC, el papel de cliente. Este modelo está soportado por la capa de aplicación COSEM y la capa de

\footnotetext{
${ }^{1}$ Simulador de eventos discretos. Información adicional en: T. Issariyakul and E. Hossain, “Introduction to Network Simulator NS2," Springer: NY, p. 435, 2009.
} 
transporte COSEM-TCP, las cuales permiten a los procesos de aplicación cliente/servidor comunicarse entre sí, por medio del intercambio de mensajes tipo request/response y a través del perfil de comunicaciones basado en TCP/IP. La Figura 3 presenta la estructura y los servicios soportados por el modelo.

La estructura, los mensajes, los servicios y los protocolos del modelo de comunicaciones DLMS/COSEM (Figura 3) se encuentran estandarizados en las normas IEC 62056-47 (capa de transporte COSEM TCP para redes IPv4) e IEC 62056-53 (capa de aplicación COSEM). Los procesos de aplicación COSEM cliente/servidor (modelo de interfaces) se describen en la norma IEC 62056-62 [6]. Este trabajo se enfocó en la parte de comunicaciones, es decir, la capa de aplicación COSEM y la capa de transporte COSEM-TCP.

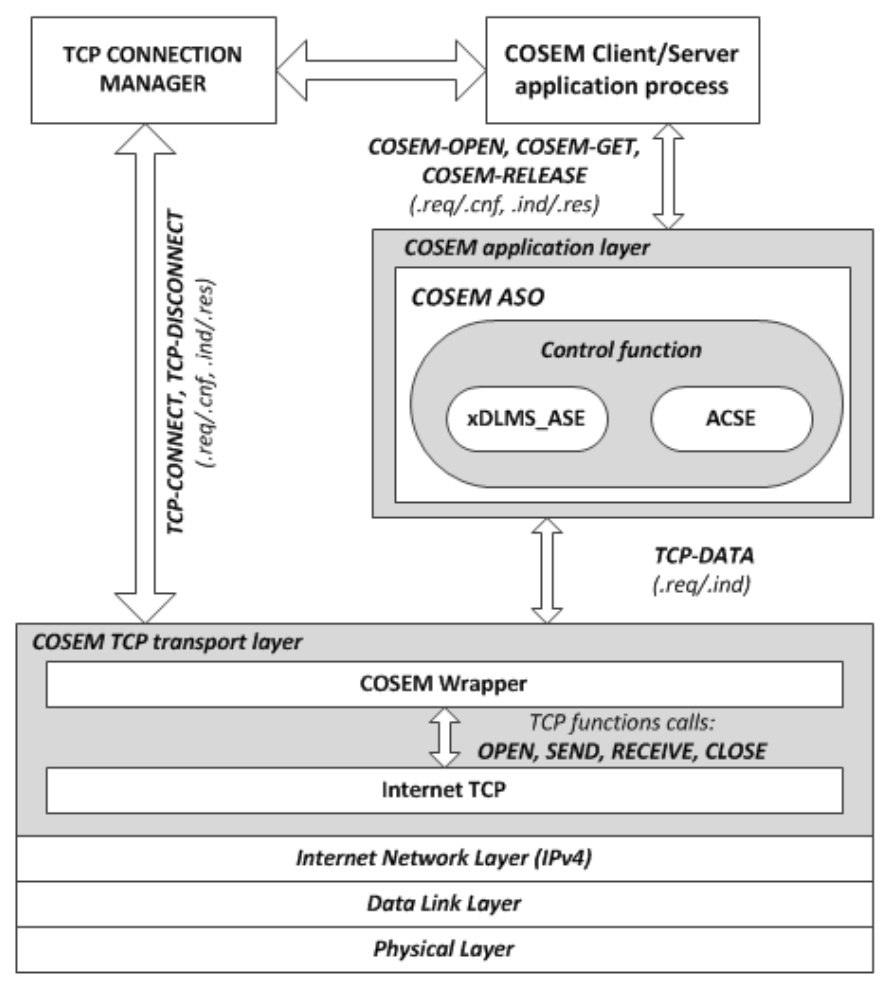

Figura 3. Estructura y servicios: modelo de comunicaciones DLMS/COSEM de los nodos Clientes/Servidor.

Con el fin de lograr la interoperabilidad entre los equipos de medición (MI) y los equipos de recolección (DC) y poder así evaluar por simulación el desempeño del esquema de comunicaciones propuesto, se realizó una extensión al simulador NS-2 mediante la adición de nuevos módulos [7], modelados con conceptos de la programación orientada a objetos (POO), tales como herencia y polimorfismo, los cuales facilitaron la codificación en $\mathrm{C}++\mathrm{y}$ permitieron aprovechar las librerías existentes NS-2. 


\subsection{Modelo de concentrador de datos}

Con el fin de no sobrecargar la red celular con el tráfico AMI y reducir así el número de accesos directos, se diseñó e implementó en C++ y se incorporó en NS-2 el componente del concentrador de datos (DC) [7]. Este dispositivo se encarga de recolectar la información generada por los MI dentro del alcance de su red (red local PLC) y de transferirlos al Centro de Control de la compañía de energía en uno o varios paquetes, reduciendo así el número de accesos directos, conexiones, señalización y overheads en la comunicación.

El diseño del componente se presenta en la Figura 4. Se compone principalmente de cuatro módulos: (1) módulo PLC, hardware NB-PLC para la comunicación con la red de medidores inteligentes; (2) módulo HSDPA, módem celular para comunicarse con el Centro de Control; (3) módulo StorageApp, encargado de gestionar la memoria del DC y los datos recibidos: permite al módulo PLC almacenar en memoria los datos capturados de la red de medidores para que el módulo HSDPA pueda hacer uso de dichos datos, los procese y los envíe por la red de acceso HSPDA y (4) módulo COSEMApp, contiene el protocolo de comunicación DLMS/COSEM, que permite el intercambio de mensajes entre el DC y los MI conectados a su red. Este último se conecta al hardware NB-PLC. Adicionalmente, el componente posee dos puntos de conexión: (1) puerto de entrada y salida PLC, al cual se conecta la línea de potencia de baja tensión perteneciente a la "última milla" (tramo que forma parte la red de medidores inteligentes) y (2) una antena $T x / R x H S D P A$, utilizada para acceder a la red celular HSDPA y permitir la comunicación con el Centro de Control.

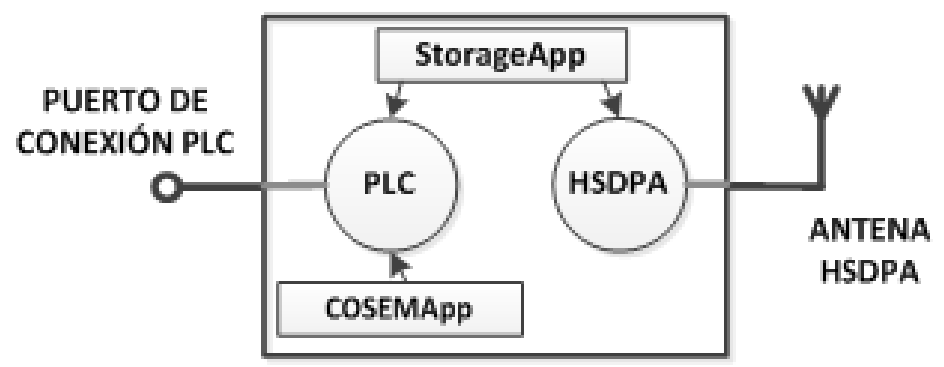

Figura 4. Arquitectura del modelo experimental de concentrador de datos (DC). Se compone de dos módulos de comunicaciones (PLC y HSDPA) y dos módulos de aplicación (COSEMAPP y StorageApp), conectados para constituir un solo nodo NS-2.

Por último, el mecanismo implementado para solicitar datos a los MI utiliza “polling” (siguiendo el estilo de un planificador Round Robin). El DC solicita las mediciones a los MI siguiendo un orden predeterminado y mantenido por éste. Una vez culminado el proceso de solicitud de datos a un MI particular, el DC solicita las mediciones al siguiente MI de la lista. Este proceso continúa hasta atender a todos los MI que se encuentran conectados a la red local gestionada por el DC. Empieza una nueva "ronda" (i.e. realiza una nueva solicitud a todos los MI) al expirar el intervalo de tiempo establecido. 


\section{Escenario y métricas de desempeño}

\subsection{Escenario}

Se propuso el escenario de simulación en NS-2 de la Figura 5 para evaluar y analizar el desempeño del esquema de comunicaciones propuesto para una red AMI. El escenario se compone de:

- Cinco redes locales de medidores inteligentes (MI), pertenecientes a una red de distribución de baja tensión, gestionadas por concentradores de datos (DC) e implementadas sobre PLC. Cada uno de los DC y los MI usa el protocolo DLMS/COSEM para establecer una comunicación con su entidad par (i.e. DC con MI y viceversa).

- Cinco concentradores de datos (DC) con capacidad de hasta 1.000 MI distribuidos sobre una zona urbana (en su mayoría residencial y comercial) y con habilidades de ejecutar mecanismos "polling" para solicitar las mediciones a los MI cada 1 minuto (tiempo coherente para aplicaciones de respuesta de la demanda). El límite del número de MI por DC se estableció teniendo en cuenta las especificaciones técnicas de diferentes DC comercialmente disponibles.

- Una micro-celda HSDPA $^{2}$ de 300 m de radio, configurada con los modelos y parámetros de la Tabla I. Tiene capacidad de atender simultáneamente a los DC y a varios usuarios móviles/fijos que demandan aplicaciones de Internet (FTP y HTTP (web)) y en tiempo real (VOIP (conversación) y Streaming (Video)), distribuidos de forma uniforme sobre el área de cobertura de la celda, dentro de una zona urbana mayoritariamente residencial y comercial, con edificios y casas de alturas uniformes por debajo de la altura de la torre celular. Los MI no necesariamente deben forman parte del área de cobertura de la celda, ya que no utilizan directamente el servicio de la red celular; por el contrario, los DC deben estar dentro del área para poder establecer una comunicación con el Centro de Control Smart Grid.

- Una red metropolitana implementada sobre fibra óptica y con capacidad de transportar el tráfico generado por los MI y los servidores.

- Cuatro servidores (VOIP, HTTP, FTP, video streaming) y el Centro de Control Smart Grid conectados a un enrutador. El Centro de Control corre la aplicación Requestin$g A P P$, encargada de solicitar y recibir los datos de los DC cada 3 minutos (intervalo adecuado para aplicaciones de respuesta a la demanda).

La pila de protocolo de las entidades principales del esquema de comunicaciones para una red AMI se detalla en la Figura 2. La configuración e implementación de los nodos que componen la red celular (Nodo B y RNC) y la red metropolitana (SGSN y GGSN) se realizó según la guía de usuario de EURANE [8] y la descripción en [9].

\footnotetext{
${ }^{2}$ Haciendo la aclaración de que sólo es posible simular con una estación base (Nodo B) en EURANE [8].
} 
Por último, se listan los supuestos realizados en la configuración de las redes locales PLC:

- Los MI se encuentran distribuidos uniformemente sobre la red local, lo cual implica que el número de MI localizados cerca del DC es menor a los que se encuentran alejados.

- Las distancias al DC (ubicado en el transformador de distribución) son menores a $200 \mathrm{~m}$. Este hecho elimina la necesidad de emplear equipos de regeneración de la señal PLC.

- Todos los MI son capaces de enviar y recibir datos directamente del DC y no establecen ninguna comunicación con sus vecinos u otro medidor fuera de la sección de red. Se conectan al DC en una topología en estrella.

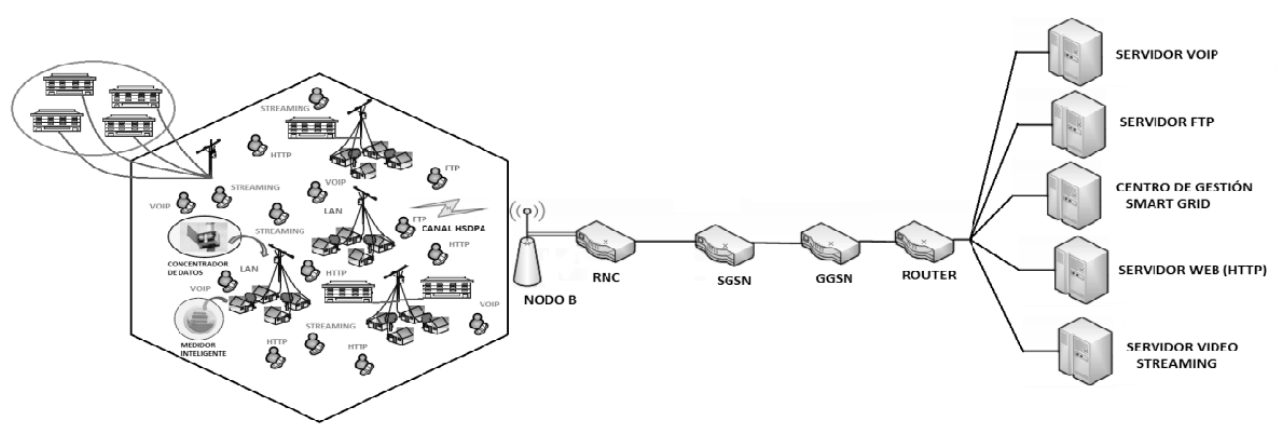

Figura 5. Escenario de simulación montado en NS-2. Se compone de 5 redes locales MIs, gestionada por 5 DCs contenidas en una micro-celda celular HSDPA de 300m de radio. Una red metropolitana sobre fibra óptica y 4 servidores de aplicaciones convencionales y el servidor del Centro de Control o Gestión Smart Grid.

Tabla I. Resumen de configuración de parámetros de la micro-celda

(Basado en [4] [10])

\begin{tabular}{cc}
\hline Configuración o Parámetros & Modelo o Valor \\
\hline Modelo de propagación & COST 231 Walfish-Ikagami \\
Pérdidas promedio de propagación $[\mathrm{dB}]$ & 112.33 \\
Radio de la celda $[\mathrm{m}]$ & 300 \\
Perfil de canal & Pedestrian A \\
Velocidad UE $[\mathrm{km} / \mathrm{h}]$ & 3 \\
RBS máximo $(\mathrm{dBm})$ & 43 \\
Ganancia de la antena $(\mathrm{dBi})$ & 17.5 \\
Correlación de distancia $(\mathrm{m})$ & 40 \\
Desviación estándar $(\mathrm{dB})$ & 8 \\
Interferencia intercelda $(\mathrm{dBm})$ & -70 \\
Interferencia intracelda $(\mathrm{dBm})$ & 30 \\
\hline
\end{tabular}




\subsection{Métricas de desempeño}

En la Tabla II se resumen las métricas de desempeño (requerimientos de calidad o QoS (Quality of Services)), los protocolos de transporte y las fuentes de tráficos para cada una de las aplicaciones ofrecidas dentro de la red AMI. Las aplicaciones de Internet (FTP \& HTTP) y en tiempo real (VOIP \& video streaming) fueron configuradas en el escenario de simulación siguiendo las recomendaciones dadas en [4] [10] [11].

Tabla II. Requerimientos de calidad para las aplicaciones ofrecidas (Basado en [11]-[15])

\begin{tabular}{|c|c|c|c|c|c|}
\hline Aplicación & VOIP & VIDEO & HTTP & FTP & AMI \\
\hline \hline End-to-End Delay & $<150 \mathrm{~ms}$ & $<250 \mathrm{~ms}$ & $<400 \mathrm{~ms}$ & No límite & $<15 \mathrm{~s}$ \\
PLR & $<10^{-2}$ & $<10^{-1}$ & $<10^{-3}$ & $<10^{-3}$ & $<10^{-3}$ \\
Jitter & $<50 \mathrm{~ms}$ & $<2 \mathrm{segs}$ & N.A. & No límite & N.A. \\
Tasa de datos (Kbps) & $4--64$ & $20--384$ & - & - & - \\
Fuente de Tráfico (NS-2) & Exp. ON/OFF & CBR & Pareto ON/OFF & FTP & DLMS/COSEM \\
\hline
\end{tabular}

\section{Resultados}

En primer lugar, se discute el impacto que genera el tráfico AMI sobre las métricas de desempeño de las aplicaciones ofrecidas en la red celular, por medio del análisis de resultados de simulación recogidos de varias corridas del escenario (con diferentes semillas), los cuales fueron procesados en Matlab. En cada corrida se incrementó el tráfico AMI enviado al Centro de Control, aumentado el número de MI atendidos por DC en cada una de las redes locales.

En segundo lugar, se analiza el desempeño de la red AMI ante el incremento en la demanda de los servicios de telefonía móvil, manteniendo fijo el número de medidores inteligentes dentro de la red PLC (número obtenido en el primer análisis).

\subsection{Tráfico en la red celular (fijo) y tráfico AMI (variable)}

La red celular transporta el tráfico generado por los servidores de aplicaciones de Internet y en tiempo real a los diferentes equipos de usuarios o UE (User Equipments) que solicitan sus servicios en la microcelda HSDPA. Adicionalmente, transporta el tráfico generado por los MI de las redes locales PLC, el cual es transferido por los nodos DC, a través de la infraestructura de telecomunicaciones hasta el Centro de Control; y por último, las solicitudes de datos emitidas por el Centro de Control a cada uno de los DCs.

Claramente, se puede observar en la Figura 6 que tanto el Throughput promedio como el retardo punto a punto promedio por aplicación se mantienen prácticamente constantes ante el incremento del número de MI por red, con variaciones muy pequeñas del orden de microsegundos y de unos cuantos bits/s, debido a la aleatoriedad introducida por el simulador NS-2 
en la generación y transmisión de paquetes. Por tanto, el tráfico AMI a través de la red celular favorece la prestación de servicios de Internet y en tiempo real con el caudal de datos suficiente por aplicación y con retardos promedios dentro de los límites establecidos por los requerimientos de calidad.

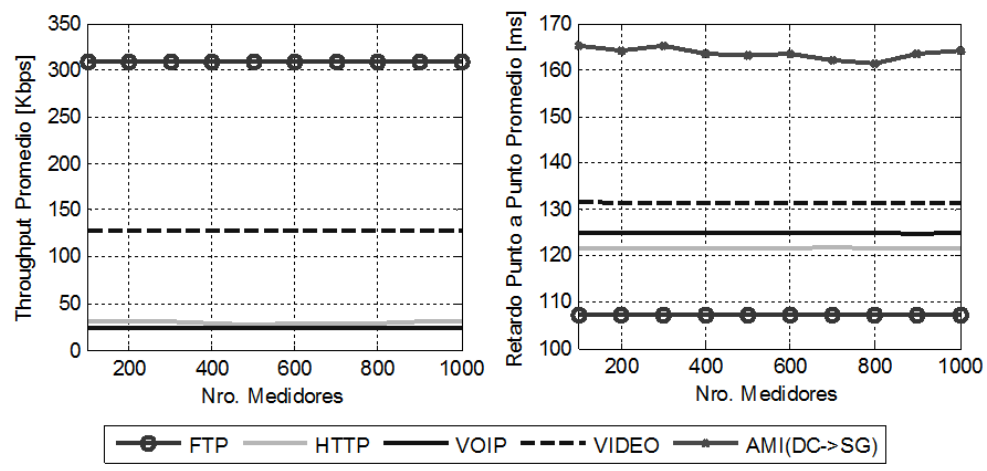

Figura 6. Throughput y retardo punto a punto promedio por aplicación. El tráfico AMI no afecta la calidad de servicios de las aplicaciones convencionales ofrecidas.

Con respecto al tráfico AMI a través de la red celular, se observa que el Throughput promedio en la dirección DC $\rightarrow$ Centro de Control (DC $\rightarrow$ SG) (Figura 7), se incrementa a medida que se aumenta el número de MI, lo cual es lógico, ya que el tráfico transferido por el DC aumenta como resultado del incremento del caudal de datos transferidos por la red de MIs. Sin embargo, está muy por debajo del Throughput promedio obtenido para las aplicaciones de Internet y en tiempo real (Figura 6). Lo anterior se debe principalmente al siguiente hecho: la cantidad de datos transferida satisfactoriamente por el DC, durante el mismo de tiempo de observación (2000s), es muy pequeña comparada con la transferida satisfactoriamente por los servidores de las aplicaciones de Internet y en tiempo real; en consecuencia, se obtiene un Throughput promedio muy bajo en relación con las demás aplicaciones. Adicionalmente, el Throughput promedio se ve afectado por los largos intervalos de tiempo establecidos para solicitar datos, de parte del DC y del Centro de Control (varios segundos en comparación con las demás aplicaciones).
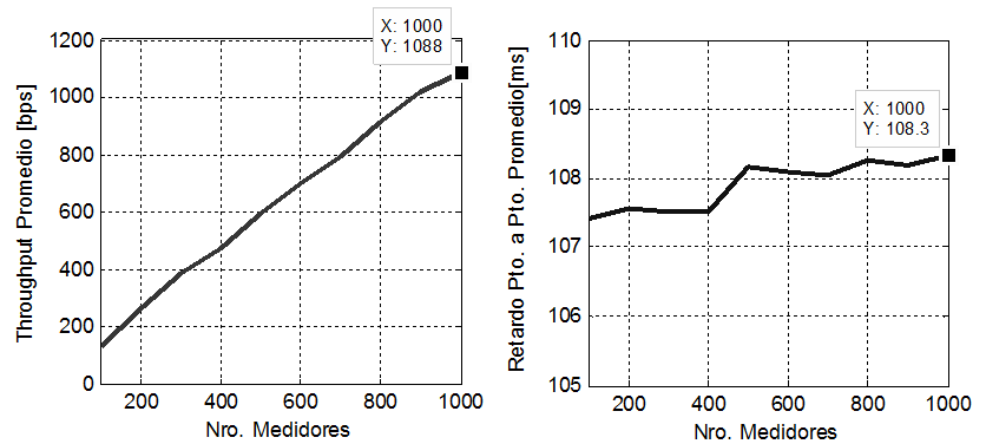

Figura 7. Métricas de desempeño del tráfico AMI sobre la red celular.

Por otro lado, se presentan mayores retardos punto a punto promedios en la transmisión de paquetes en la dirección SG $\rightarrow$ DC (Figura 6) comparada con la dirección DC $\rightarrow$ SG 
(Figura 7). Las diferencias principales entre las dos direcciones están en el canal físico y en el protocolo de transporte empleado: en la dirección SG $\rightarrow$ DC se emplea el canal compartido HS-DSCH (High Speed-Downlink Shared Channel) y los paquetes son transferidos utilizando el protocolo de transporte no orientado a la conexión, UDP; mientras que en la dirección DC $\rightarrow$ SG se emplea el canal dedicado DPDCH (Dedicated Physical Data Channel) y el protocolo orientado a la conexión, TCP. Los altos retardos promedios presentados en la dirección SG $\rightarrow$ DC pueden ser ocasionados por los retardos introducidos en las colas de PDU (Protocol Data Units) dentro del transmisor del Nodo B (i.e. se transmiten los PDU en el siguiente $\mathrm{TTI}^{3}$ ) y por el hecho de utilizar un canal compartido por todos los UE.

Por otro lado, el retardo total AMI promedio (Figura 8), es decir, el tiempo empleado desde el momento que se emite la solicitud por parte del Centro de Control hasta que éste recibe los datos transferidos por el DC, es de 3,089 s, intervalo durante el cual se transfieren 26,37 Kbytes en promedio en cada solicitud realizada por el Centro (para un total de 1.000 MI por DC). Este retardo está por debajo del límite establecido por los QoS (Tabla II).
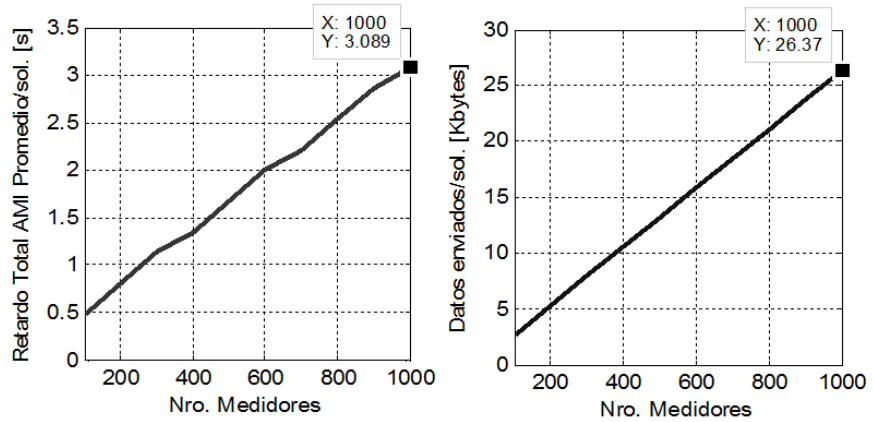

Figura 8. Retardo total promedio y tráfico AMI transferido al Centro.

Tabla III. Tabla comparativa de desempeño entre redes AMI implementadas sobre PLC + HSPA y HSDPA (Basado en [11]-[15])

\begin{tabular}{|c|c|c|c|}
\hline Métricas & Aplicación & 5000 MIs (PLC + HSDPA) & 130 MIs (HSDPA)[4] $^{1}$ \\
\hline \hline \multirow{4}{*}{$\begin{array}{c}\text { Retardo punto a punto } \\
\text { promedio [ms] }\end{array}$} & FTP & 107,1 & 346 \\
\cline { 2 - 4 } & HTTP & 121,5 & 235 \\
\cline { 2 - 4 } & VOIP & 125 & 127 \\
\cline { 2 - 4 } & VIDEO & 131,3 & 231 \\
\cline { 2 - 4 } & AMI(DC $\rightarrow$ SG) & 108,3 & 245 \\
\cline { 2 - 4 } & AMI(SG $\rightarrow$ DC) & 164,1 & - \\
\hline \multirow{4}{*}{$\begin{array}{c}\text { Porcentaje de paquetes } \\
\text { recibidos promedio [ \%] }\end{array}$} & FTP & 99,99 & 99,99 \\
\cline { 2 - 4 } & HTTP & 99,99 & 99,19 \\
\cline { 2 - 4 } & VOIP & 99,99 & 98,66 \\
\cline { 2 - 4 } & VIDEO & 99,99 & 99,11 \\
\cline { 2 - 4 } & AMI & 100 & 99,99 \\
\hline
\end{tabular}

Por último, en la Tabla III se confrontan los resultados de desempeño obtenidos para el es-

\footnotetext{
${ }^{3}$ Transmission Time Interval. En HSDPA es igual a 2 ms.
} 
quema de comunicaciones propuesto en este artículo con el esquema propuesto por los autores en [4]. Ambos esquemas utilizan el protocolo DLMS/COSEM para solicitar las mediciones y configuraciones similares en la microcelda HSDPA (Tabla I). Se puede observar que el esquema propuesto en este artículo presenta un desempeño superior al propuesto en [4], en cuanto a retardos punto a punto, lo cual da garantía de una alta calidad en la prestación de servicios de aplicaciones de Internet y en tiempo real. Además, presenta retardos DC $\rightarrow \mathrm{SG}$ de 108,3 ms en la transmisión de bloques de datos AMI (1040 Bytes) y ofrece una alta confiabilidad, por encima del 99,9\% en todas las aplicaciones. Por tanto, bajo este esquema, se logra alto porcentaje de cobertura en número de clientes para la compañía de energía y se reduce el número de accesos a la red celular, dado que acceden únicamente los 5 DC y no los 5.000 MI, lo que permite no sobrecargar la red y garantizar la calidad en los servicios actualmente prestados en la microcelda HSDPA. Este hecho puede motivar a los operadores de telefonía a ofrecer sus servicios de infraestructuras de telecomunicaciones a los operadores de red que los requieran.

\subsection{Tráfico en la red celular (variable) y tráfico AMI (fijo)}

Dado que el tráfico AMI generado por 5.000 medidores inteligentes no deterioró la calidad de los servicios de telefonía móvil prestados en la microcelda HSDPA, se analizaron escenarios con microceldas de mayor capacidad en número de usuarios ${ }^{4}$, manteniendo fijo el tráfico AMI. Se asumió que dentro de la microcelda predominan los usuarios que demandan servicios de VOIP y navegación por la web (HTTP).

Se derivó una carga de tráfico de aplicaciones de Internet y en tiempo real correspondiente a la carga de una celda de mayor capacidad de usuarios. Sin comprometer la exactitud de los resultados de simulación, se asumió que el tráfico recibido por un usuario (User Equipment) dentro de la microcelda, equivale al tráfico que recibirían simultáneamente múltiples usuarios que demandan los servicios de Internet y en tiempo real. Este hecho asume que los usuarios se encuentran ubicados sobre las mismas coordenadas en el escenario de simulación. En escenarios reales, se traduciría en múltiples usuarios muy cerca unos de otros.

La Figura 9 muestra los resultados de simulación obtenidos para diferentes cargas de tráfico de aplicaciones de telefonía móvil. Se escogieron las métricas y las aplicaciones mostradas en la figura, ya que fueron las más afectadas en las diferentes corridas de simulación realizadas.

Claramente se observa que las métricas de desempeño para las aplicaciones de video y $R e$ questingApp (más específicamente, las solicitudes generadas por el Centro de Control, SG $\rightarrow$ DC), se deterioran a medida que se incrementa el tráfico dentro de la microcelda, llegando hasta el punto en que ya no es posible garantizar los requerimientos de calidad para el servicio de video. Con 426 usuarios dentro de la microcelda, se obtuvo un retardo punto a punto promedio superior a $250 \mathrm{~ms}$ (i.e. no se satisface los QoS establecidos (Tabla II)) y un porcentaje de paquetes recibidos del $97,68 \%$, es decir, la confiabilidad se redujo un 2,31\% comparado con la confiabilidad obtenida en el análisis anterior (Tabla III). Por tanto, al no garantizar los requerimientos de calidad de servicio (QoS) de las aplicaciones de telefonía, ya no es posible seguir atendiendo a los 5.000 MI. Adicionalmente, el tiempo en que se demoran en llegar las

\footnotetext{
${ }^{4}$ UEs que demandan simultáneamente los servicios de Internet y en tiempo real. EURANE soporta únicamente 20 nodos UEs simultáneamente dentro una celda celular.
} 

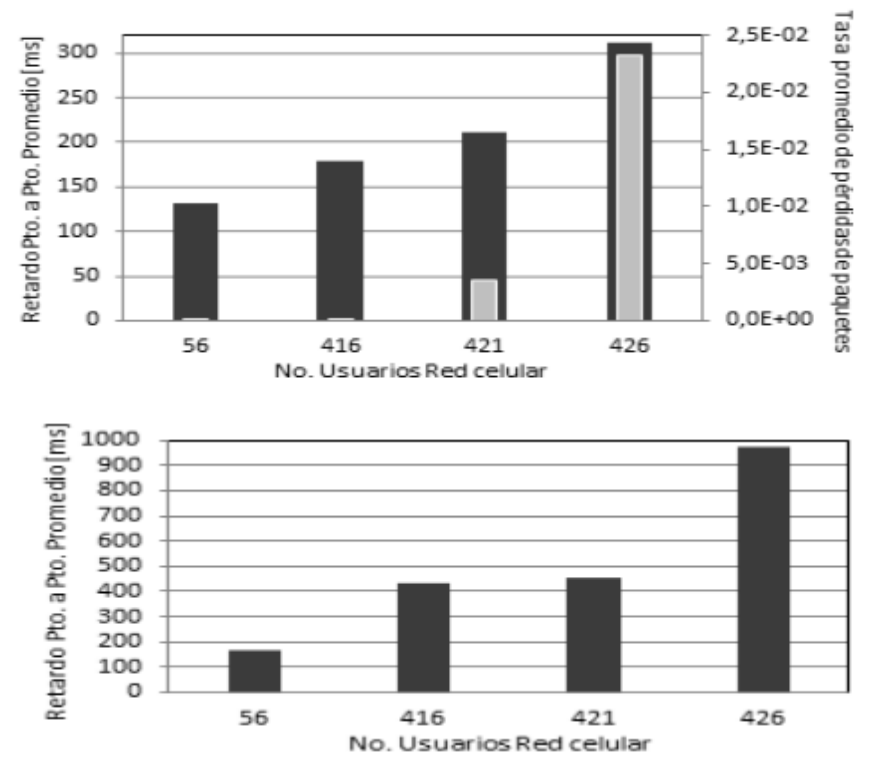

Figura 9. Métricas de desempeño obtenidas al variar el tráfico de las aplicaciones de telefonía móvil. (a) Aplicación de video (streaming): barra negra, tasa promedio de pérdidas de paquetes; barra gris, retardo punto a punto promedio (ms). (b) Aplicación RequestingAPP.

solicitudes generadas por el Centro a los DC ubicados al borde de la celda, se incrementó de $164,1 \mathrm{~ms}$ a $0,970 \mathrm{~s}$.

A partir del escenario con 426 usuarios simultáneos atendidos por la estación base ubicada dentro de la microcelda, se realizaron varios ensayos de simulación para determinar el número de MI por red local capaz de soportar la red AMI sin afectar los requerimientos de calidad de las demás aplicaciones. Luego de variar el número de MI por red local, realizando múltiples corridas de simulación, se determinó que con 275 MI por red local se logra prestar todos los servicios de Internet y en tiempo real, y se garantizan los requerimientos de calidad de servicio (QoS) establecidos. Este número sigue siendo favorable, comparado con los $130 \mathrm{MI}$ logrados con el esquema AMI propuesto en [4], para el operador de red que desee desplegar una red AMI en sectores de menor capacidad de clientes.

\section{Conclusiones y trabajo futuro}

En este artículo se presentaron los resultados del diseño y evaluación del desempeño de un esquema de comunicaciones para una red AMI, usando el simulador de redes NS-2. El esquema combinó las tecnologías PLC (Power Line Communication) y HSDPA (High Speed Downlin Packet Access), así como el uso de concentradores de datos y de protocolos como DLMS/COSEM y TCP-UDP/IP.

Se extendió el simulador NS-2 introduciendo los módulos: (1) Protocolo DLMS/COSEM de acuerdo con las normas IEC 62056-47 (capa de transporte) e IEC 62056-53 (capa de aplicación) y (2) el componente concentrador de datos (DC). 
Los resultados numéricos obtenidos indican que al introducir el concentrador de datos (DC) dentro de la AMI, la red celular, operando bajo condiciones normales, presenta un desempeño superior frente a un esquema que no emplea DC para concentrar la información de diferentes redes de medidores inteligentes (MI) (Tabla III). Adicionalmente, al considerar los DC dentro del esquema AMI se logra mayor cobertura y menor número de accesos directos a la red celular (únicamente acceden a la red 5 DC con capacidad de atender $1.000 \mathrm{MI}$, sin sacrificar la calidad en los servicios prestados). Sin embargo, para escenarios con mayor números de usuarios simultáneos dentro de la microcelda, se pudo observar que los requerimientos de calidad de las aplicaciones de Internet y en tiempo real, especialmente de los servicios de video (streaming), se vieron afectados, lo que implicó reducir el número de MI atendidos por red local (de 1.000 a $275 \mathrm{MI}$ ), con el fin de garantizar la calidad de los servicios prestados.

Como trabajo futuro se propone realizar la validación rigurosa del módulo protocolo DLMS/COSEM por medio de mediciones reales, haciendo uso de equipos de medición y concentración de datos reales.

\section{References}

[1] J. Wang and V. Leung, “A Survey of Technical Requirements and Consumer Application Standards for IP-based Smart Grid AMI Network,” in 2011 Int. Conf. Information Networking (ICOIN), pp. 114-119.

[2] H. Sui, H. Wang, M. Lu, and W. Lee, “An AMI System for the Deregulated Electricity Markets," IEEE Trans. on Industry Applications, vol. 45, no. 6, pp. 2104-2108, December 2009.

[3] A. Zaballos, "Survey and Performance Comparison of AMR over PLC Standards", IEEE Transactions of Power Delivery, vol. 24, No 2, pp. 604-613, April 2009.

[4] A. M. Ruíz y H. G. Narváez, "Evaluación de desempeño de una red de medidores inteligentes, implementada sobre tecnología celular HSDPA,” Tesis de Magíster, Departamento de Ingeniería Eléctrica y Electrónica, Universidad de los Andes, Bogotá, pp. 1-154, 2011.

[5] High-level Smart Meter Data Traffic Analysis, Engage Consulting, United Kingdom, May 2010. Available on: www.engage-consulting.co.uk

[6] Electricity metering-data exchange for meter reading, tariff and load control: IEC-62056 Parts 47 and 53, IEC, 2006.

[7] J. M. Aranda, "Evaluación del desempeño de una red AMI implementada sobre las tecnologías PLC y HSDPA,” Bogotá: Tesis Magíster, Universidad de los Andes, 2012, pp. 1-148.

[8] EURANE [Online]. Available on: http://yotrew.blogspot.com/2014/01/patch-ns2-to-support-umts.html

[9] A. Alexiou, C. Bouras, and V. Igglesis (2007, March). Performance Evaluation of TCP over UMTS Transport Channels [Online]. Research Unit 6 (RU6) of Computer Technology Institute \& Press “Diophantus", University of Patras. Available on: http://ru6.cti.gr/ru6/publications/

[10] I. de BRUIN et al., "Performance Analysis of Hybrid-ARQ Characteristics in HSDPA," Wireless Personal Communications, Springer, 2007, pp. 337-353.

[11] R. Shreevastav, C. McGoldrick, and M. Huggard, “Delivering Improved QoS and Cell Throughput in UMTS Based HSDPA Networks," in 2009 IEEE International Symposium on a Word of Wireless, Mobile and Multimedia Networks \& Workshops, pp. 1-9.

[12] A. Brkanic, A, M. Hadzialic. M, and D. Borovina, "Effects of Choice of MAC Protocol on QoS Parameters in BPL Network,” in 2008 International Symposium ELMAR-2008, Croatia, pp. 285-288.

[13] US Department of Energy (October, 2010). Report: Communications Requirements for Smart Grid Technology [Online]. Available on: http://energy.gov/sites/prod/files/gcprod/documents/

[14] 3GPP TS 22.105 Technical Specification, 3GPP, V8.4.0, June 2006.

[15] ITU-T G.1050, Telecommunication Standardization Sector of ITU, November 2007. 


\section{Juan Manuel Aranda L. K.}

Nació en Ciudad del Este, Paraguay. Es Ingeniero Electrónico de la Universidad de los Andes, de Bogotá, Colombia. Obtuvo su título de Maestría en Ingeniería en la Universidad de los Andes, de Bogotá, Colombia. Actualmente se desempeña como docente investigador en el área de Telemática en la Universidad Sergio Arboleda de Bogotá, Colombia.

e-mail: juan.aranda@usa.edu.co 